\title{
CROP SUITABILITY ANALYSIS FOR CEREAL CROPS OF UTTAR PRADESH, INDIA
}

\author{
Pragati Singh ${ }^{1, *}$, R. K. Upadhyay ${ }^{1}$, Hiren P. Bhatt ${ }^{2}$, Markand P. Oza ${ }^{2}$, S. P. Vyas ${ }^{2}$ \\ ${ }^{1}$ Remote Sensing Applications Centre, Uttar Pradesh, India - (singh22pragati, rsacupard) @ gmail.com \\ ${ }^{2}$ Space Applications Centre (ISRO), India - (hiren, markand, spvyas) @ sac.isro.gov.in
}

\section{Commission V, SS: Natural Resources Management}

KEY WORDS: Agriculture, AHP Method, Analysis, Suitability, Soil Moisture, Temporal, Weightage.

\begin{abstract}
:
The crop suitability is the process of assessing the appropriateness or ability of a given type of land on the basis of growing conditions of a particular crop. The study focused on the crop suitability analysis of cereal crops for their production in Uttar Pradesh. Information about crop suitability is essential for proper management of agriculture in the study area. Remote sensing and GIS data provide a reliable information and technique to find suitable land for crops. The research was based on GIS based MultiCriteria Decision Approach. The AMSR-2 (Advance Microwave Scanning Radiometer) soil moisture data, Carto-DEM, soil chemical and physical properties and climate data were used to identify the crop suitability in the study area. Weightage of different factors was arrived at based on input and feedback from experts. An Analytical Hierarchical Process (AHP) was used in ArcGIS environment to generate suitability map for the cereals crop. The suitability map has been categorised in the form of highly suitable, moderately suitable, low suitable and non-agricultural/ non-suitable region of the study area for Wheat, Rice, Sorghum, Maize and Pearl Millet/Bajra.
\end{abstract}

The overall study indicates that the study area has a huge potential of cereal crop production. Therefore, improved levels of agricultural production can be achieved by cultivating crop in highly and moderately suitable areas; and practicing diversification of marginally suitable areas to crops other than that for which it is low suitable.

\section{INTRODUCTION}

Agriculture is one of the most important sectors for India. It is necessary for our country to arrange enough food for the people of our country (Patel and Oza, 2014). Among all types of crops, Cereals are the major food crops. They provide adequate food calories and about half of the protein requirement of population. Cereals constitute staple food in the diet. The cereal grain contains on an average $58-72 \%$ carbohydrates, $8-13 \%$ protein, $2-5 \%$ fat and $2-10 \%$ indigestible fibre (NBSSLUP 2004). It plays a significant role to satisfy hunger for most of the population. To increase the production of cereals, besides proper planning of land, relevant, reliable and timely information is required to arrive at most suitable sites for their cultivation."

The crop suitability analysis is the process of assessing the appropriateness or ability of a given type of land on the basis of growing conditions of a particular crop. The suitability is a function of crop requirements and land characteristics. It is a measure of how well the qualities of land unit match with the requirements of a particular form of land use (FAO, 1976).

Remote sensing and GIS data provide a reliable information and technique to find the suitable land for crops, which saves resources time and money and provides reliable information to farmers and policy makers for enhancing the production and reducing the cost. The research was based on GIS based MultiCriteria Decision Approach, which uses information on several variables related to crop requirement, so that conducive land for cereal crop growth can be found out by assigning appropriate importance to those layers in weighted overlay technique of

\footnotetext{
* Corresponding author
}

spatial analyst tool, according to the requirement of particular cereals.

\section{STUDY AREA \& DATA SETS USED}

The study area was taken as whole Uttar Pradesh state of India (Figure 1).With the total area of 2,187, $28.25 \mathrm{~km}^{2}$, and situated between $23^{\circ} 52^{\prime} \mathrm{N}$ and $31^{\circ} 28^{\prime} \mathrm{N}$ latitudes and $77^{\circ} 3^{\prime}$ and $84^{\circ} 39^{\prime} \mathrm{E}$ longitudes, is the fourth largest state of India. It plays a significant role in the contribution of national food grain stock. Agriculture is the major industry of the state and is source of livelihood for 72 percent of its population. Uttar Pradesh is the major producer of cereals in India. Its economy is basically based on the agricultural productions.

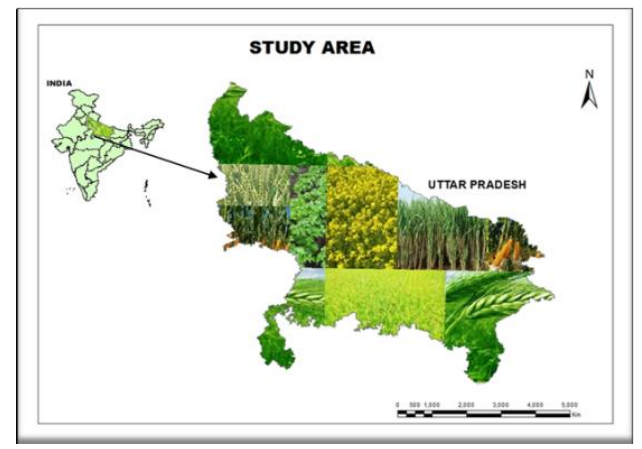

Figure 1. Study area (Uttar Pradesh)

\begin{tabular}{|c|c|c|c|c|}
\hline Data & $\begin{array}{c}\text { Acquis } \\
\text { ition } \\
\text { Year }\end{array}$ & Source & $\begin{array}{c}\text { Scale/ } \\
\text { Spatial } \\
\text { Resolution }\end{array}$ & Bands/Description \\
\hline AMSR2 & $\begin{array}{c}2012- \\
2017\end{array}$ & JAXA & $\begin{array}{c}1: 1,000,000 / \\
10 \mathrm{~km}\end{array}$ & L \\
\hline
\end{tabular}




\begin{tabular}{|c|c|c|c|c|}
\hline CARTO-DEM & & $\begin{array}{l}\text { SDIS (VEDAS/ } \\
\text { SAC) }\end{array}$ & $\begin{array}{c}1: 1,000 / \\
10 \mathrm{~m}\end{array}$ & PAN \\
\hline $\begin{array}{l}\text { Land Use and } \\
\text { Land Cover } \\
\text { Map }\end{array}$ & 2012 & RSAC (U.P.) & $1: 50,000$ & $\begin{array}{l}\text { Built-up lands, Wet-lands, } \\
\text { Water bodies, Forest, Barren } \\
\text { lands, rock out-crops, Waste } \\
\text { lands, Agriculture lands, } \\
\text { Grass land, Semi grass lands }\end{array}$ \\
\hline Rainfall & $\begin{array}{ll}1980- \\
2015\end{array}$ & IMD & $0.25^{0} \times 0.25^{0}$ & Daily rainfall data in $\mathrm{cm}$ \\
\hline $\begin{array}{c}\text { Temperature } \\
\text { data }\end{array}$ & $\begin{array}{ll}1980- \\
2015\end{array}$ & IMD & $1^{0} \times 1^{0}$ & $\begin{array}{c}\text { Daily temperature data in } \\
\text { degree centigrade }\end{array}$ \\
\hline \begin{tabular}{|c|} 
Soil \\
Characteristic \\
s (texture, \\
depth, \\
productivity, \\
erosion)
\end{tabular} & 2006 & NNRMS & $1: 50,000$ & $\begin{array}{l}\text { Soil type, depth in } \mathrm{cm} \text {, high } \\
\text { to low productivity areas and } \\
\text { deep to shallow erosion areas }\end{array}$ \\
\hline N.P.K. map & 2011- & $\begin{array}{c}\text { Soil testing } \\
\text { Laboratory } \\
\text { Agriculture } \\
\text { Department } \\
\text { Lucknow }\end{array}$ & 1:150,000 & $\begin{array}{c}\text { fertility map has given the } \\
\text { value of Nitrogen, Potash and } \\
\text { Phosphate }\end{array}$ \\
\hline $\begin{array}{l}\text { Bio climatic } \\
\text { zone map }\end{array}$ & 2011 & NBSSLUP & $1: 50,000$ & $\begin{array}{l}\text { It has three humid, sub } \\
\text { humid and semi dry bio- } \\
\text { climatic region }\end{array}$ \\
\hline $\begin{array}{c}\text { Length of } \\
\text { growing } \\
\text { period }\end{array}$ & 2011 & NBSSLUP & $1: 50,000$ & $\begin{array}{l}120 \text { to } 240 \text { days area wise } \\
\text { length of growing period }\end{array}$ \\
\hline Soil pH map & 2004 & NBSSLUP & $1: 50,000$ & \begin{tabular}{|c|} 
It has area wise soil $\mathrm{pH}$ value \\
of Uttar Pradesh from 4.5 to \\
$>9.5$
\end{tabular} \\
\hline Drainage map & 2004 & NBSSLUP & $1: 50,000$ & $\begin{array}{c}\text { Well drain to poorly drain } \\
\text { areas map }\end{array}$ \\
\hline $\begin{array}{c}\text { Salinity } \\
\text { \&Sodicity }\end{array}$ & 2004 & NBSSLUP & $1: 50,000$ & $\begin{array}{l}\text { It has Sodic and Saline area } \\
\text { ranges from normal to high }\end{array}$ \\
\hline
\end{tabular}

Table 1. Description of datasets used

\subsection{AMSR2 Soil Moisture Data}

Advance Microwave Scanning Radiometer (AMSR) Soil Moisture $10 \mathrm{~km}$ spatial resolution product has been downloaded from Jaxa Aerospace agency website from May 2012 to March 2017. The data set is passive microwave L band frequency data, there are two data sets available daily for AMSR2soil moisture data product; one is for ascending pass (during day time) another is for descending pass (during night time) which covers India in two days.

\subsection{Carto DEM}

Carto DEM data has been taken from the Satellite Data Information System (SDIS; VEDAS/SAC). This is $10 \mathrm{~m}$ panchromatic data set for Uttar Pradesh, which is used for creating Slope Map of Uttar Pradesh.

\subsection{Land use and Land cover Map}

Land Use land cover map for the Uttar Pradesh has been taken from Remote Sensing Applications Centre Uttar Pradesh which is available for the Year 2012. It was used to differentiate the agricultural and non-agricultural areas.

\subsection{Rainfall and Temperature Data}

Rainfall and temperature data has been taken from repository of Space Applications Centre Ahemdabad (reference: ncc@imd.gov.in; National Climate Centre India Meteorological Department). The data is in the Gridded form which is available on the daily basis from 1980 to 2015 . Spatial resolution of Rainfall data is $0.25^{0} \times 0.25^{0}$ and for Temperature is $1^{0} \times 1^{0}$. It was used for required climatic conditions for crops in the study area.

2.5 Soil Characteristics (Texture, Depth, Productivity and Erosion)
The soil characteristics map has been taken from NNRMS website (www.nnrms.gov.in; National Natural Management System) in Shape file format at 1:50,000 scale. All these map consist different classes (Table 1). The soil texture map has been further reclassified on the basis of family of particle size using Canadian (Canadian Agriculture and Agri-food department) Soil classifications.

\subsection{N.P.K. Map}

N.P.K. map has been taken from the Soil Testing Laboratory of Agricultural Department Lucknow for the year 2011-2012 in the form of image file on the scale of 1:150,000. The map has been scanned on high resolution then it was georeferenced taking more than 250 reference points with 0.015 RMS errors on first order polynomial, after that it was digitized and classified on the basis of map legends.

\subsection{BCL and LGP Map}

Bioclimatic zone map (BCL) and Length of Growing Period (LGP) map has been taken from NBSSLUP (National Bureau of Soil Survey and Land use Planning) from Agro Ecological Zone of Uttar Pradesh for the year 2011 on the scale of 1:50,000, in the form of Image File which is further digitized same as N.P.K map has been.

\subsection{Soil pH, Drainage, Salinity \& Sodicity Map}

Soil $\mathrm{pH}$, Drainage, Salinity \& Sodicity map has been taken from NBSSLUP (National Bureau of Soil Survey and Land use Planning) from Soil characteristics of Uttar Pradesh for the year 2004 on the scale of 1:50,000, in the form of Image File which is further digitized same as N.P.K map has been.

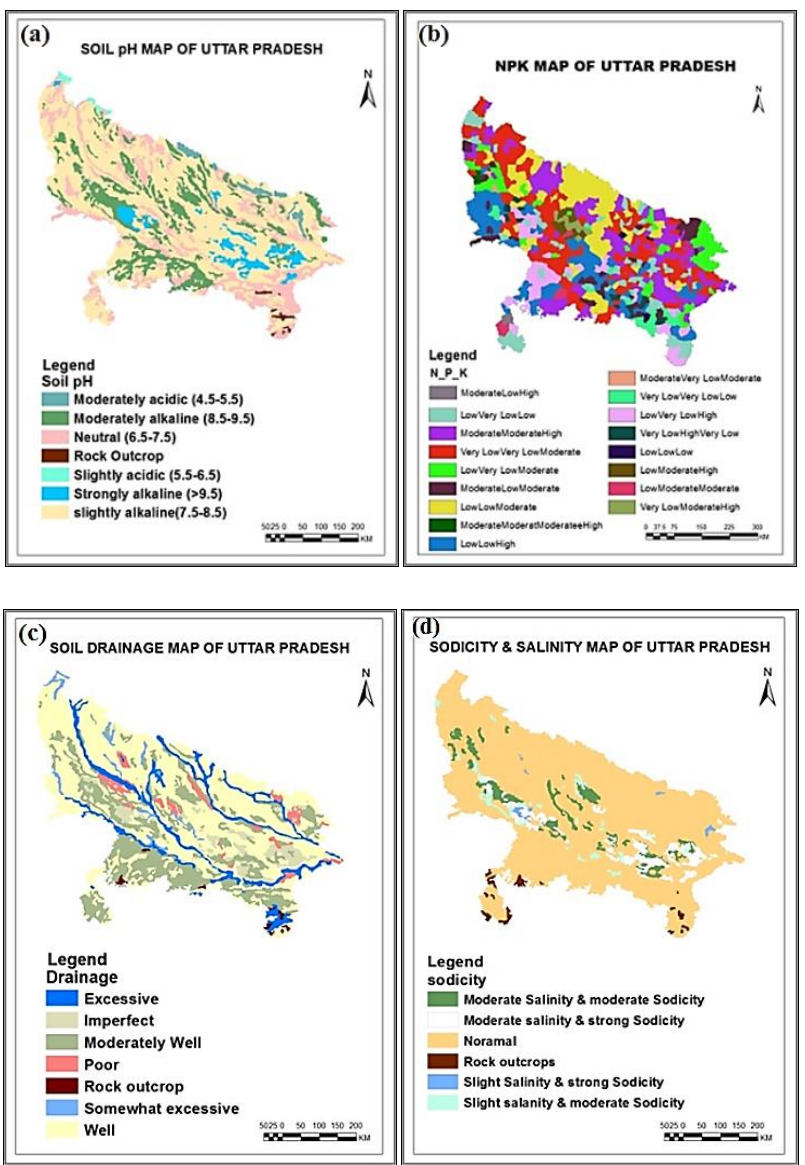



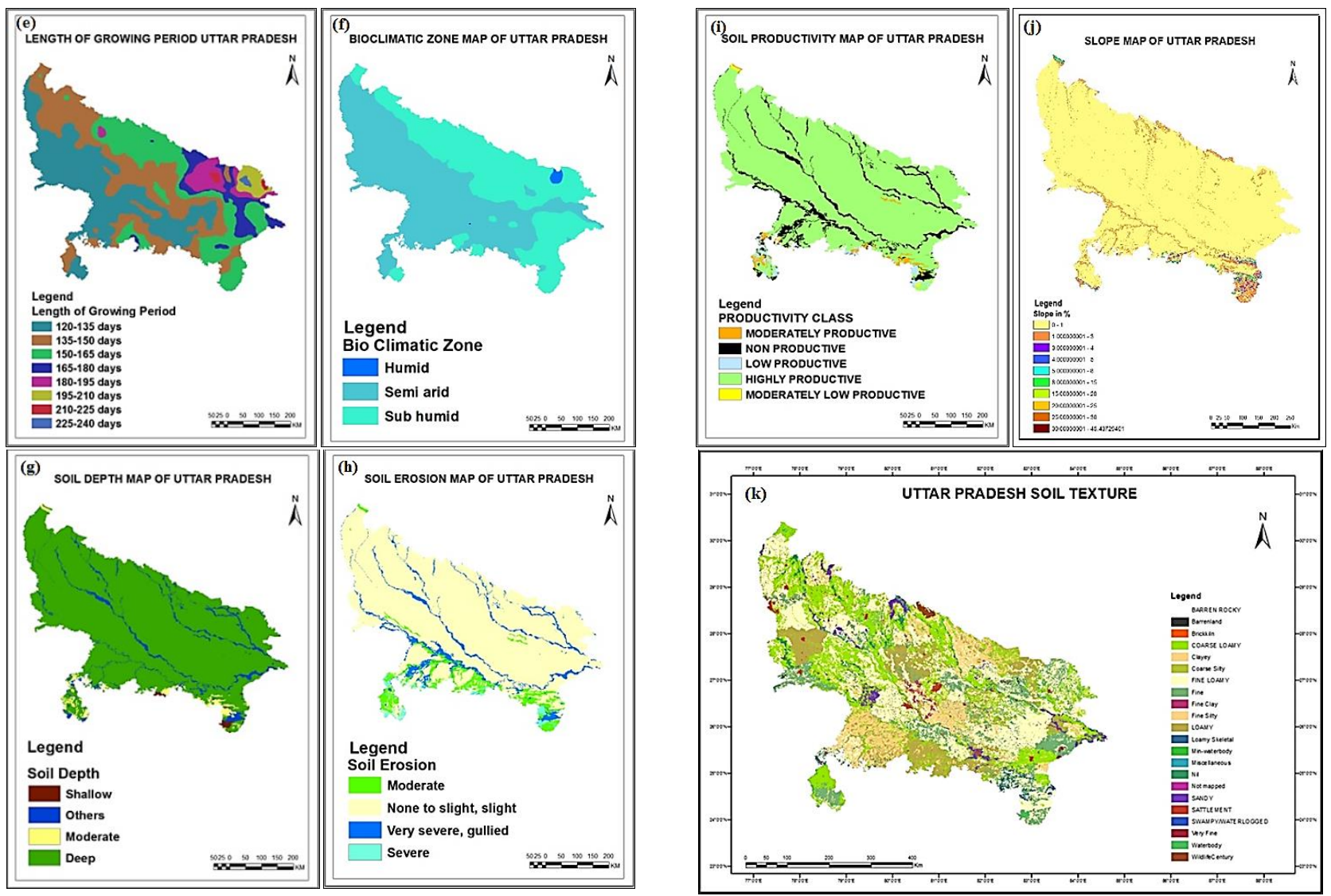

Figure 2. Input maps of Uttar Pradesh (a) soil pH, (b) NPK, (c) drainage, (d) Sodicity \& salinity, (e) Length of growing period, (f) Bioclimatic region, (g) Soil depth, (h) Soil erosion, (i) Soil productivity, (j) slope, (k) soil texture

\begin{tabular}{|c|c|c|c|c|c|}
\hline $\begin{array}{l}\text { Classes of } \\
\text { Parameters }\end{array}$ & AREA (ba) & Classes of Parameters & AREA (ha) & Classes of Parameters & AREA (ha) \\
\hline Soil Erosion & & Soil Depth & & N.P.K & \\
\hline Moderate & $14,978,00.00$ & Others & $12,173,25.00$ & Low, Very Low, Low & $17,417,80.00$ \\
\hline None to slight, slight & $184,545,00.00$ & Maderate & $4,677,50.00$ & Moderate, Moderate, High & $35,751,30.00$ \\
\hline \multirow[t]{2}{*}{ Severe } & $3,296,75.00$ & Shallow & $859,50.00$ & Low, Very Low, Moderate & $15,341,50.00$ \\
\hline & & & & Moderate, Low, Moderate & $9,637,75.00$ \\
\hline Soil Drainage & & Soil Texture & & Low, Low, Moderate & $31,958,00.00$ \\
\hline Exceasive & $20,286,20.00$ & Water body & $22,093,30.00$ & Moderate, Very Low, Moderate & $425,00.00$ \\
\hline Rock autcrop & $1,206,50.00$ & Sandy clay loam & $254,213, \infty 0.00$ & Very Law, Very Low, Law & $5,070,00.00$ \\
\hline Somewhat exceagive & $4,227,00.00$ & Miscellameons & $122,00.00$ & Low, Very Low, High & $15,390,80.00$ \\
\hline Imperfect & $12,165,00,00$ & Loamy gand & $8,402,25.00$ & Very Law, High, Very Low & $2,848,25.00$ \\
\hline \multirow[t]{2}{*}{ Poor } & $7,810,25.00$ & Silty loam & $92,969,00.00$ & Low, Low, Law & $813,25.00$ \\
\hline & & SATTLEMIENT & $11,737,50.00$ & Low, Moderate, High & $2,725,75.00$ \\
\hline $\begin{array}{l}\text { Length of } \\
\text { Growing period }\end{array}$ & & BARREN ROCKY & $447,25.60$ & Low, Moderate, Moderate & $2,182,75.00$ \\
\hline $210-225$ days & $1,006,50.00$ & Silty & $8,736,50.00$ & MODERATELY PRODUCTIVE & $3,046,50.00$ \\
\hline $180-195$ days & $10,062,00.00$ & Heaxy Clay & $435,00.00$ & NON PRODUCTTE & $17,191,50.00$ \\
\hline $225-240$ days & $615,00.00$ & Silty Clay & $2,979,75.00$ & DATA NOT AVAIIABLE & $17,25.00$ \\
\hline \multirow[t]{2}{*}{$195-210$ days } & $5,038,75.00$ & Brick kiln & $66,00.00$ & LOW PRODUCTTVE & $3,224,25.00$ \\
\hline & & Nil & $7,734,75,00$ & HIGHIY PRODUCTIVE & $194,675,00.00$ \\
\hline Soil pH & & Barren land & $46,00.00$ & MODERATELY LOW PRODUCTIVE & $652,50.00$ \\
\hline Neutral $(6.5-3.5)$ & $43,410,50.00$ & Wild life Century & $2,340,00.00$ & & \\
\hline $\begin{array}{l}\text { slightly alkaline(7.5- } \\
\text { 8.5) }\end{array}$ & $122,484,00.00$ & SWAMIPY/WATERLOGGED & $48,50.00$ & Sodicity\& Salinity & \\
\hline $\begin{array}{l}\text { Moderately alkaline } \\
(8.5-9.5)\end{array}$ & $36,852,50.00$ & & & Slight Salinity \& moderate Sodicity & $4,877,75.00$ \\
\hline Strongly alkaline & $10,080,30.00$ & Bio Climatic rone & & Moderate Salinity \& moderate Sodicity & $11,960,50.00$ \\
\hline $\begin{array}{l}\text { Moderately acidic } \\
\langle 4.5-5.5\rangle\end{array}$ & $3,796,00.00$ & Semi-arid & $123,745,00.000$ & Moderate Salinity \& strong Sodicity & $12,297,30.00$ \\
\hline
\end{tabular}

Table 2. Area of parameters classes (ha) 


\section{METHODOLOGY}

\subsection{AMSR2 Soil Moisture Data}

AMSR2 soil moisture data has been daily averaged removing no data, cloud and water body value, after that weekly average map has been prepared from July 2012 to April 2017. After making average the pixel size of soil moisture data had been resample using bilinear interpolation and at last the soil moisture data for each and every week according to the crop duration and moisture requirement has been classified as; normal moisture at showing time high at growing period and low at harvesting time. Then Create single image for each and every crop using weighted sum.

\subsection{Climate data processing}

Climate is the result of 30 to 35 years average of rainfall and temperature for any particular place. The rainfall data is taken from IMD from 1980 to 2016 on the scale of $0.25^{\circ} \times 0.25^{\circ}$ and the temperature data is from 1980 to 2015 on the scale of $1^{0} \times 1^{0}$ (source: Space Applications Centre Ahemdabad \& reference: ncc@imd.gov.in (National Climate Centre India Meteorological Department)). First of all monthly sum of rainfall data and average of temperature data from 1980-2016 for each month by daily data has been created, then the average map of 37 years for rainfall and temperature has been generated. After the generation of rainfall and temperature map the pixel size was resample using bilinear interpolation technique. Then it was classified according to crop requirements.

\subsection{GIS Data preparation:}

The remote sensing image can't provide all the parameters for crop suitability analysis. That's why the ancillary data has been also taken for generating data base for crop suitability analysis. The reference layers has been Georeferenced, and then digitized all the parameters of image. After the digitization vector files were converted into raster format and then it was classified according to NBSSLUP guidelines.

\subsection{Selection and Classification of Parameters}

There are number of parameters has been taken for cereals crop suitability analysis of Uttar Pradesh. The parameters were selected using NBSSLUP guideline for soil site requirement for crops. The categories have been classified into four parts as: S1 for highly suitable, S2 for moderately suitable, S3 for low suitable and $\mathrm{N}$ for not suitable using NBSSLUP guidelines. "Table 5" represents the NBSSLUP guideline for cereals crops;

\subsection{Multi-Criteria Decision making (MCDM) approach and Analytical Hierarchical Process (AHP)}

Analytical Hierarchy Process (AHP) is one of Multi Criteria basic leadership technique that was created by Professor Thomas L. Saaty's. The AHP is a decision making technique which can be utilized to take care of complex choice issues. The information is inferred by utilizing an arrangement of combine shrewd correlations. These examinations are utilized to acquire the weights of significance of the choice criteria, and the relative execution me*asures of the choices as far as every individual choice basis. To put it plainly, it is a strategy to get proportion scales from combined examinations.

At more elevated amounts of the chain of command the criteria are required to be assessed to infer the weights. Here the criteria weights should be summed up to 1 , so the entrenched geometric mean technique is utilized. In this approach every one of the components in the line are duplicated and the nth root is ascertained and are separated by their whole to get the standardized weights Table 3 .

\begin{tabular}{|c|c|c|}
\hline $\begin{array}{c}\text { Intensity of } \\
\text { Importance }\end{array}$ & Definition & Explanation \\
\hline 1 & Equal importance & $\begin{array}{c}\text { Two elements contribute equally to } \\
\text { the property }\end{array}$ \\
\hline 3 & $\begin{array}{c}\text { Moderate importance } \\
\text { of one over another }\end{array}$ & $\begin{array}{c}\text { Experience and judgment slightly } \\
\text { favour one over the other }\end{array}$ \\
\hline 5 & $\begin{array}{c}\text { Essential or strong } \\
\text { importance }\end{array}$ & $\begin{array}{c}\text { Experience and judgment strongly } \\
\text { favour one over another }\end{array}$ \\
\hline 9 & $\begin{array}{c}\text { Very strong } \\
\text { importance }\end{array}$ & $\begin{array}{c}\text { An element is strongly favoured } \\
\text { and its dominance is demonstrated } \\
\text { in practice }\end{array}$ \\
\hline $2,4,6,8$ & $\begin{array}{c}\text { Intermediate values } \\
\text { between two adjacent } \\
\text { judgments }\end{array}$ & $\begin{array}{c}\text { The evidence favouring one } \\
\text { Comprise is needed between two } \\
\text { judgments }\end{array}$ \\
\hline
\end{tabular}

Table 3. Saaty's Ratio scale for pair wise comparison of importance of weights of criteria

Considering the "Table 3" the following formula was used to implement the AHP technique to assign the weightage of crops;

$$
\begin{aligned}
& \text { 1. Step }=\frac{n(n-1)}{2} \quad \text { 3. Step }= \\
& \text { 2. Step }=\mathrm{A}=\left(\begin{array}{cccc}
a_{i i} & a_{i j} & \ldots & a_{i n} \\
a_{j i} & a_{j j} & \ldots & a_{j n} \\
\ldots & \ldots & \ldots & \ldots \\
a_{i n} & a_{j n} & \ldots & a_{m n}
\end{array}\right) \quad \quad a_{i j}=\frac{1}{a_{j i}} \\
& \text { For all } \quad t=1,2, \ldots \ldots, n \text { and } j=1,2 \text {, } \\
& \text { 4. Step }= \\
& \overline{a_{i j}}=\frac{a_{i j}}{\sum_{i=1}^{n} a_{i j}} \\
& \text { For all } j=1,2, \ldots . ., n \\
& \text { 5. Step }= \\
& W_{i}=\frac{\sum_{j=1}^{n} \overline{a_{i j}}}{n} \\
& \text { For all } i=1,2 \ldots n \\
& \text { 6. Step }=C I=\frac{\left(\lambda_{\max }-n\right)}{(n-1)} \\
& \text { Where, } \mathrm{CI}=\text { Consistency Index, } \\
& \text { 7. Step }=\quad C R=\frac{C I}{R I}
\end{aligned}
$$

\begin{tabular}{|l|l|l|l|l|l|l|l|l|l|l|}
\hline $\mathrm{n}$ & 1 & 2 & 3 & 4 & 5 & 6 & 7 & 8 & 9 & 10 \\
\hline $\mathrm{Rl}$ & 0 & 0 & 0.58 & 0.9 & 1.12 & 1.24 & 1.32 & 1.41 & 1.45 & 1.49 \\
\hline
\end{tabular}

Table 4. Random Consistency Index (RI)

Following the above formula of Saaty's pair wise comparison matrix for calculating the weightage of parameters according to their priorities, the weightage for cereal crops has been calculated in the following "table no. 6, 7, 8, 9 and 10". 


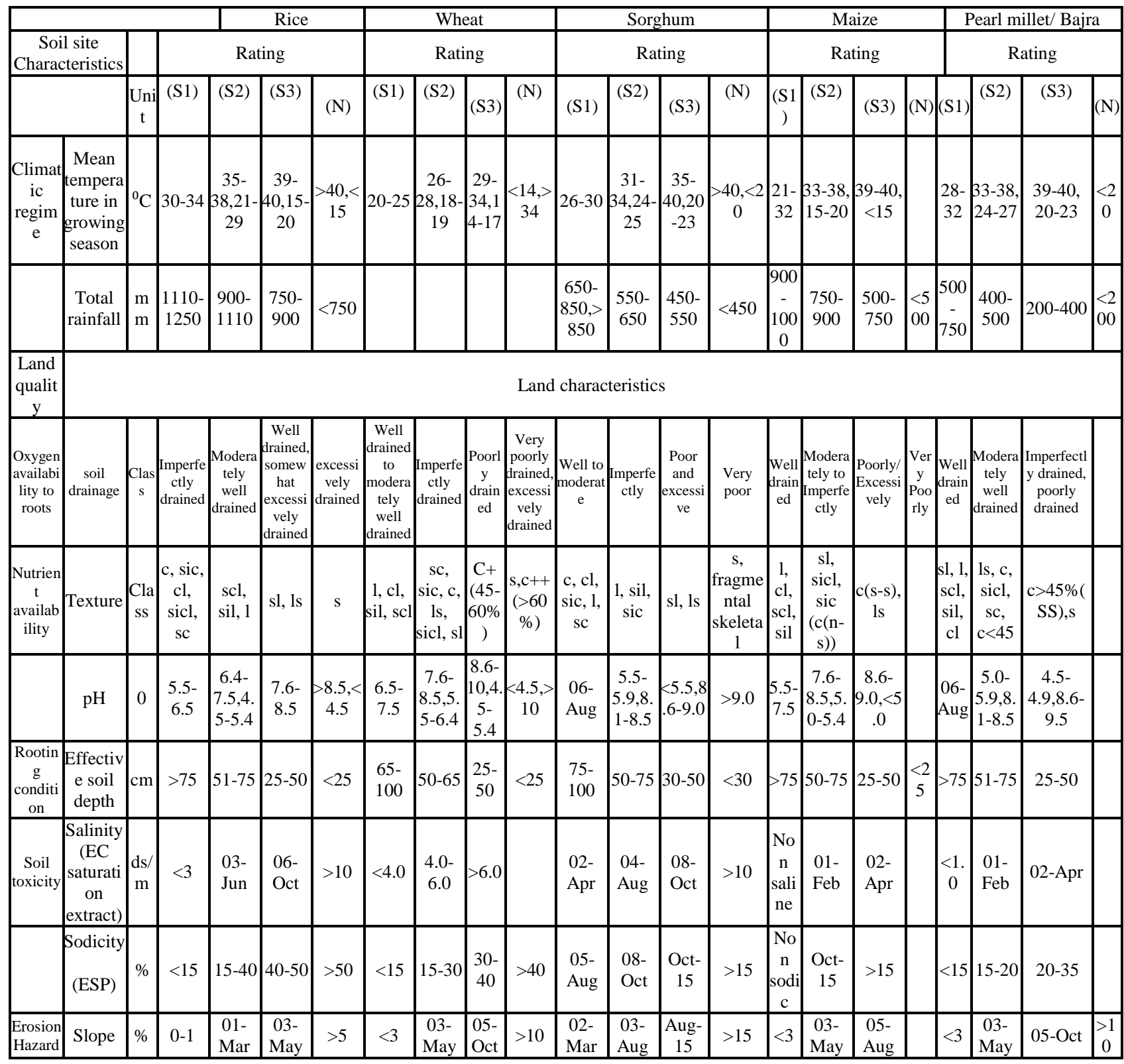

*S1- highly suitable, S2- moderately suitable, S3- Low suitable, S1-Not suitable, S- Sand, C- clay, sc- Sandy clay, scl- Sandy clay loam, sic- Silty clay, sicl- Silty clay learn, sil- Silt loam, sl- Sandy loam, ls- Loamy sand, 1- Loam, EC- Electrical conductivity, clClay loam, C(n-s)- Non swelling clay, C (ss)- Shrink-swell clay,

Table 5. Soil site requirement for Cereals (NBSSLUP)

\begin{tabular}{|c|c|c|c|c|c|c|c|c|c|}
\hline $\begin{array}{c}\text { Parame } \\
\text { ter }\end{array}$ & $\begin{array}{l}\text { SMC } \\
\& \\
\text { rainf } \\
\text { all }\end{array}$ & $\begin{array}{c}\text { Slope, } \\
\text { draina } \\
\text { ge \& } \\
\text { erosion }\end{array}$ & \begin{tabular}{|c|} 
soil \\
textu \\
re \\
$\&$ \\
$\operatorname{dept}$ \\
h \\
\end{tabular} & \begin{tabular}{|c|}
$\mathbf{B C}$ \\
$\mathbf{L}^{*}$ \\
$\boldsymbol{\&}$ \\
$\mathbf{L G}$ \\
$\mathbf{P}^{*}$ \\
\end{tabular} & $\begin{array}{c}\text { NPK }{ }^{*} \& \\
\text { Producti } \\
\text { vity }\end{array}$ & $\begin{array}{c}\text { Temper } \\
\text { ature }\end{array}$ & pH & $\begin{array}{c}\text { Sodici } \\
\text { ty } \\
\& \\
\text { Salini } \\
\text { ty }\end{array}$ & $\begin{array}{c}\text { Weight } \\
\text { age }\end{array}$ \\
\hline $\begin{array}{l}\text { SMC\& } \\
\text { rainfall }\end{array}$ & 1.00 & 3.00 & 7.00 & 3.00 & 5.00 & 7.00 & $\begin{array}{c}7.0 \\
0 \\
\end{array}$ & 7.00 & 0.35 \\
\hline $\begin{array}{c}\text { Slope, } \\
\text { drainag } \\
\text { e \& } \\
\text { erosion } \\
\end{array}$ & 0.33 & 1.00 & 5.00 . & 3.00 & 3.00 & 5.00 & $\begin{array}{c}3.0 \\
0\end{array}$ & 5.00 & 0.20 \\
\hline $\begin{array}{c}\text { soil } \\
\text { texture } \\
\text { \&depth }\end{array}$ & 0.14 & 0.20 & 1.00 & 0.20 & 1.00 & 3.00 & $\begin{array}{c}2.0 \\
0\end{array}$ & 3.00 & 0.07 \\
\hline BCL \& & 0.33 & 0.33 & 5.00 & 1.00 & 3.00 & 5.00 & 1.0 & 3.00 & 0.13 \\
\hline
\end{tabular}

\begin{tabular}{|c|c|c|c|c|c|c|c|c|c|}
\hline LGP & & & & & & 0 & & \\
\hline $\begin{array}{c}\text { NPK \& } \\
\text { Product } \\
\text { ivity }\end{array}$ & 0.20 & 0.33 & 1.00 & 0.33 & 1.00 & 3.00 & $\begin{array}{c}3.0 \\
0\end{array}$ & 5.00 & 0.09 \\
\hline $\begin{array}{c}\text { Temper } \\
\text { ature }\end{array}$ & 0.14 & 0.20 & 0.33 & 0.20 & 1.00 & 1.00 & $\begin{array}{c}0.3 \\
3\end{array}$ & 5.00 & 0.05 \\
\hline pH & 0.14 & 0.33 & 0.50 & 1.00 & 0.33 & 3.00 & $\begin{array}{c}1.0 \\
0\end{array}$ & 3.00 & 0.07 \\
\hline $\begin{array}{c}\text { Sodicity } \\
\& \\
\text { Salinity }\end{array}$ & 0.14 & 0.20 & 0.33 & 0.33 & 0.20 & 0.20 & $\begin{array}{c}0.3 \\
3\end{array}$ & 1.00 & 0.03 \\
\hline \begin{tabular}{c}
$\lambda_{\max }=8.14$ \\
\hline
\end{tabular} & $\mathrm{CI}=0.02$ & $\begin{array}{c}\mathrm{CR}= \\
0.014\end{array}$ & \multicolumn{6}{|c|}{ Total=1.00 } \\
\hline
\end{tabular}

Table 6. Pairwise comparison matrix of selected criteria's and their weights for Rice 


\begin{tabular}{|c|c|c|c|c|c|c|c|c|c|}
\hline $\begin{array}{c}\text { Paramet } \\
\text { ers }\end{array}$ & $\begin{array}{c}\text { Soil } \\
\text { textur } \\
\text { e \& } \\
\text { depth }\end{array}$ & $\left|\begin{array}{c}\text { SMC }^{*} \\
\& \\
\text { rainfal } \\
1\end{array}\right|$ & $\begin{array}{c}\text { Temperat } \\
\text { ure }\end{array}$ & \begin{tabular}{|c|} 
Slope, \\
draina \\
ge \& \\
erosio \\
n
\end{tabular} & $\begin{array}{c}\text { NPK \& } \\
\text { productiv } \\
\text { ity }\end{array}$ & $\left|\begin{array}{c}\text { Soi } \\
1 \\
\text { pH }\end{array}\right|$ & $\mid \begin{array}{c}\text { BC } \\
\mathbf{L}^{*} \mathbf{A} \\
\mathbf{L G} \\
\mathbf{P}^{*}\end{array}$ & $\mid \begin{array}{c}\text { Sodicit } \\
\text { y\& } \\
\text { Salinit } \\
\mathrm{y}\end{array}$ & $\underset{\mathbf{e}}{\text { Weightag }}$ \\
\hline $\begin{array}{c}\text { Soil } \\
\text { texture } \\
\& \text { depth }\end{array}$ & 1.00 & 3.00 & 0.33 & 5.00 & 3.00 & $\begin{array}{c}1.0 \\
0\end{array}$ & 1.00 & 3.00 & 0.17 \\
\hline $\begin{array}{l}\text { SMC \& } \\
\text { rainfall }\end{array}$ & 0.33 & 1.00 & 0.33 & 3.00 & 1.00 & $\begin{array}{c}1.0 \\
0\end{array}$ & 1.00 & 1.00 & 0.10 \\
\hline $\begin{array}{c}\text { Tempera } \\
\text { ture }\end{array}$ & 3.00 & 3.00 & 1.00 & 5.00 & 3.00 & $\begin{array}{c}1.0 \\
0\end{array}$ & 3.00 & 3.00 & 0.25 \\
\hline \begin{tabular}{|c|} 
Slope, \\
drainage \\
$\&$ \\
erosion \\
\end{tabular} & 0.20 & 0.33 & 0.20 & 1.00 & 1.00 & $\begin{array}{c}1.0 \\
0\end{array} \mid$ & 0.33 & 3.00 & 0.07 \\
\hline \begin{tabular}{|c|}
$\begin{array}{c}\text { NPK \& } \\
\text { producti } \\
\text { vity }\end{array}$ \\
\end{tabular} & 0.33 & 1.00 & 0.33 & 1.00 & 1.00 & $\begin{array}{c}1.0 \\
0\end{array}$ & 1.00 & 3.00 & 0.09 \\
\hline Soil pH & 1.00 & 1.00 & 1.00 & 1.00 & 1.00 & $\begin{array}{c}1.0 \\
0 \\
\end{array}$ & 1.00 & 3.00 & 0.13 \\
\hline $\begin{array}{c}\text { BCL \& } \\
\text { LGP }\end{array}$ & 1.00 & 1.00 & 0.33 & 3.00 & 1.00 & $\begin{array}{c}1.0 \\
0 \\
\end{array}$ & 1.00 & 7.00 & 0.14 \\
\hline $\begin{array}{c}\text { Sodicity } \\
\& \\
\text { Salinity }\end{array}$ & 0.33 & 1.00 & 0.33 & 0.33 & 0.33 & $\left|\begin{array}{c}0.3 \\
3\end{array}\right|$ & 0.14 & 1.00 & 0.05 \\
\hline$\lambda_{\max }=8$ & & \multicolumn{2}{|c|}{$\mathrm{CI}=\mathbf{0 . 1 3}$} & $\begin{array}{l}\text { CR= } \\
0.09\end{array}$ & & & & & \\
\hline
\end{tabular}

Table 7. Pairwise comparison matrix of selected criteria's and their weights for Wheat

\begin{tabular}{|c|c|c|c|c|c|c|c|c|c|}
\hline $\begin{array}{c}\text { Paramet } \\
\text { ers }\end{array}$ & \begin{tabular}{|c|} 
Soil \\
textur \\
e \& \\
depth
\end{tabular} & $\left|\begin{array}{c}\text { SMC }^{*} \\
\& \\
\text { rainfa } \\
\text { II }\end{array}\right|$ & $\begin{array}{c}\text { Temperat } \\
\text { ure }\end{array}$ & \begin{tabular}{|c|} 
Slope, \\
draina \\
ge \& \\
erosio \\
n \\
\end{tabular} & $\begin{array}{c}\text { NPK }^{*} \& \\
\text { productivi } \\
\text { ty }\end{array}$ & $\begin{array}{l}\text { Soil } \\
\text { pH }\end{array}$ & \begin{tabular}{|l|}
$\mathbf{B C}$ \\
$\mathbf{L}^{*}$ \\
$\boldsymbol{\&}$ \\
$\mathbf{L G}$ \\
$\mathbf{P}^{*}$ \\
\end{tabular} & \begin{tabular}{|c|} 
Sodici \\
ty\& \\
Salinit \\
$y$ \\
\end{tabular} & Weighta \\
\hline \begin{tabular}{|c|} 
Soil \\
texture \\
\& depth
\end{tabular} & 1.00 & 0.33 & 7.00 & 5.00 & 1.00 & $|1.00|$ & 3.00 & 1.00 & 0.16 \\
\hline $\begin{array}{l}\text { SMC \& } \\
\text { rainfall }\end{array}$ & 3.00 & 1.00 & 3.00 & 7.00 & 3.00 & $|0.33|$ & 5.00 & 0.33 & 0.18 \\
\hline $\begin{array}{c}\text { Temper } \\
\text { ature }\end{array}$ & \begin{tabular}{|l|l|} 
\\
\end{tabular} & 0.33 & 1.00 & 3.00 & 0.33 & $|0.33|$ & 1.00 & 0.20 & 0.05 \\
\hline \begin{tabular}{|c|} 
Slope, \\
drainag \\
e \& \\
erosion \\
\end{tabular} & 0.20 & 0.14 & 0.33 & 1.00 & 0.33 & 0.20 & 0.33 & 0.11 & 0.02 \\
\hline \begin{tabular}{|c|c} 
NPK \& \\
producti \\
vity \\
\end{tabular} & 1.00 & 0.33 & 3.00 & 3.00 & 1.00 & 1.00 & 3.00 & 0.20 & 0.10 \\
\hline Soil pH & $|1.00|$ & 3.00 & 3.00 & 5.00 & 1.00 & 1.00 & 3.00 & 1.00 & 0.18 \\
\hline $\begin{array}{c}\text { BCL \& } \\
\text { LGP }\end{array}$ & 0.33 & 0.20 & 1.00 & 3.00 & 0.33 & 0.33 & 1.00 & 0.14 & 0.05 \\
\hline \begin{tabular}{|c} 
Sodicity \\
$\&$ \\
Salinity
\end{tabular} & 1.00 & 3.00 & 5.00 & 9.00 & 5.00 & 1.00 & 7.00 & 1.00 & 0.26 \\
\hline$\lambda_{\max }=8$ & 8.96 & \multicolumn{2}{|c|}{$\mathrm{CI}=0.13$} & \multicolumn{2}{|c|}{ CR $=0.09$} & & & \multicolumn{2}{|c|}{ Total $=1$} \\
\hline
\end{tabular}

Table 8. Pairwise comparison matrix of selected criteria's and their weights for Sorghum

\begin{tabular}{|c|c|c|c|c|c|c|c|c|c|}
\hline $\begin{array}{c}\text { Parameter } \\
\text { S }\end{array}$ & $\begin{array}{c}\text { Soil } \\
\text { textu } \\
\text { re \& } \\
\text { depth }\end{array}$ & $\begin{array}{c}\text { SMC } \\
\text { rainfa } \\
\text { II }\end{array}$ & $\begin{array}{c}\text { Temperat } \\
\text { ure }\end{array}$ & $\begin{array}{c}\text { Slope, } \\
\text { draina } \\
\text { ge \& } \\
\text { erosio } \\
\text { n }\end{array}$ & $\begin{array}{c}\text { NPK* } \& \\
\text { productiv } \\
\text { ity }\end{array}$ & $\begin{array}{c}\text { Soi } \\
\text { I }\end{array}$ & $\begin{array}{c}\text { BCL } \\
\text { pH }\end{array}$ & $\begin{array}{c}\text { Sodicity } \\
\text { LGP }\end{array}$ & $\begin{array}{c}\text { Salinity } \\
\text { Saighta }\end{array}$ \\
\hline $\begin{array}{c}\text { Soil } \\
\text { texture \& } \\
\text { depth }\end{array}$ & 1.00 & 5.00 & 0.33 & 5.00 & 0.33 & $\begin{array}{c}1.0 \\
0\end{array}$ & 1.00 & 0.33 & 0.11 \\
\hline $\begin{array}{c}\text { SMC \& } \\
\text { rainfall }\end{array}$ & 0.20 & 1.00 & 1.00 & 3.00 & 0.33 & $\begin{array}{c}0.3 \\
3\end{array}$ & 1.00 & 0.33 & 0.08 \\
\hline
\end{tabular}

\begin{tabular}{|c|c|c|c|c|c|c|c|c|c|}
\hline $\begin{array}{c}\text { Temperat } \\
\text { ure }\end{array}$ & 3.00 & 1.00 & 1.00 & 5.00 & 1.00 & $\begin{array}{c}7.0 \\
0\end{array}$ & 5.00 & 5.00 & 0.27 \\
\hline $\begin{array}{c}\text { Slope, } \\
\text { drainage } \\
\& \text { erosion }\end{array}$ & 0.20 & 0.33 & 0.20 & 1.00 & 0.33 & $\begin{array}{c}1.0 \\
0\end{array}$ & 0.33 & 1.00 & 0.05 \\
\hline $\begin{array}{c}\text { NPK \& } \\
\text { productivi } \\
\text { ty }\end{array}$ & 3.00 & 3.00 & 1.00 & 3.00 & 1.00 & $\begin{array}{c}3.0 \\
0\end{array}$ & 5.00 & 3.00 & 0.22 \\
\hline Soil pH & 1.00 & 3.00 & 0.14 & 1.00 & 0.33 & $\begin{array}{c}1.0 \\
0\end{array}$ & 3.00 & 1.00 & 0.09 \\
\hline $\begin{array}{c}\text { BCL \& } \\
\text { LGP } \\
\end{array}$ & 1.00 & 1.00 & 0.20 & 3.00 & 0.20 & $\begin{array}{c}0.3 \\
3 \\
\end{array}$ & 1.00 & 3.00 & 0.08 \\
\hline $\begin{array}{c}\text { Sodicity \& } \\
\text { Salinity }\end{array}$ & 3.00 & 3.00 & 0.20 & 1.00 & 0.33 & $\begin{array}{c}1.0 \\
0\end{array}$ & 0.33 & 1.00 & 0.09 \\
\hline \multicolumn{2}{|l|}{$\lambda_{\max }=$} & \multicolumn{2}{|c|}{$\mathrm{CI}=0.13$} & \multicolumn{2}{|c|}{ CR= 0.09} & & & \\
\hline
\end{tabular}

Table 9. Pairwise comparison matrix of selected criteria's and their weights for Maize

\begin{tabular}{|c|c|c|c|c|c|c|c|c|c|}
\hline$\underset{\text { rs }}{\text { Paramete }}$ & \begin{tabular}{|c} 
Soil \\
textu \\
re \& \\
dept \\
h
\end{tabular} & $\begin{array}{c}\text { SMC } \\
{ }_{*}^{*} \& \\
\text { rainf } \\
\text { all }\end{array}$ & $\begin{array}{c}\text { Temperat } \\
\text { ure }\end{array}$ & \begin{tabular}{|c|} 
Slope, \\
draina \\
ge \& \\
erosio \\
n
\end{tabular} & $\begin{array}{c}\text { NPK } \& \\
\text { producti } \\
\text { vity }\end{array}$ & \begin{tabular}{|c|} 
So \\
il \\
p \\
H
\end{tabular} & $\begin{array}{c}\text { BCL } \\
\& \\
\text { LGP }\end{array}$ & {$\left[\begin{array}{c}\text { Sodicit } \\
y \& \\
\text { Salinit } \\
y\end{array}\right.$} & $\begin{array}{c}\text { Weight } \\
\text { age }\end{array}$ \\
\hline \begin{tabular}{|c|} 
Soil \\
texture \& \\
depth \\
\end{tabular} & 1.00 & 1.00 & 1.00 & 3.00 & 1.00 & $\begin{array}{c}3.0 \\
0\end{array}$ & 3.00 & 3.00 & 1.00 \\
\hline $\begin{array}{l}\text { SMC \& } \\
\text { rainfall }\end{array}$ & 1.00 & 1.00 & 0.33 & 1.00 & 1.00 & $\begin{array}{c}3.0 \\
0 \\
\end{array}$ & 5.00 & 3.00 & 1.00 \\
\hline $\begin{array}{c}\text { Temperat } \\
\text { ure }\end{array}$ & 1.00 & 3.00 & 1.00 & 3.00 & 1.00 & $\begin{array}{c}5.0 \\
0\end{array}$ & 7.00 & 3.00 & 1.00 \\
\hline \begin{tabular}{|c} 
Slope, \\
drainage \\
\& erosion
\end{tabular} & 0.33 & 1.00 & 0.33 & 1.00 & 0.33 & $\begin{array}{c}3.0 \\
\text { 3 }\end{array}$ & 5.00 & 3.00 & 0.33 \\
\hline \begin{tabular}{|c|c|} 
NPK \& \\
productiv \\
ity
\end{tabular} & 1.00 & 1.00 & 1.00 & 3.00 & 1.00 & $\begin{array}{c}3.0 \\
0\end{array}$ & 7.00 & 3.00 & 1.00 \\
\hline Soil pH & 0.33 & 0.33 & 0.20 & 0.33 & 0.33 & $\begin{array}{c}1.0 \\
0\end{array}$ & 1.00 & 1.00 & 0.33 \\
\hline $\begin{array}{c}\text { BCL \& } \\
\text { LGP }\end{array}$ & 0.33 & 0.20 & 0.14 & 0.20 & 0.14 & $\begin{array}{c}1.0 \\
0\end{array}$ & 1.00 & 1.00 & 0.33 \\
\hline $\begin{array}{c}\text { Sodicity } \\
\& \\
\text { Salinity }\end{array}$ & 0.33 & 0.33 & 0.33 & 0.33 & 0.33 & $\begin{array}{c}1.0 \\
0\end{array}$ & $\mid 1.00$ & 1.00 & 0.33 \\
\hline$\Lambda \max =$ & 8.46 & \multicolumn{2}{|c|}{$\mathrm{CI}=0.07$} & \multicolumn{2}{|c|}{$\mathrm{CR}=0.05$} & & & \multicolumn{2}{|c|}{ Total $=1$} \\
\hline
\end{tabular}

Table 10. Pairwise comparison matrix of selected criteria's and their weights for Pearl millet

*SMC- soil moisture content, NPK- nitrogen-phosphate-potash,

BCL- bio climatic region, LGP- length of growing period.

By using the above weightage in the weighted overlay tool in ArcGIS, Cereal crop suitability output has been generated. After the generation of output it has been mask out by land use land cover mask of Uttar Pradesh to exclude the non-agricultural region. And final cereal suitability map has been generated. The flow chart (fig: 3) represents the methodology followed in this work. 


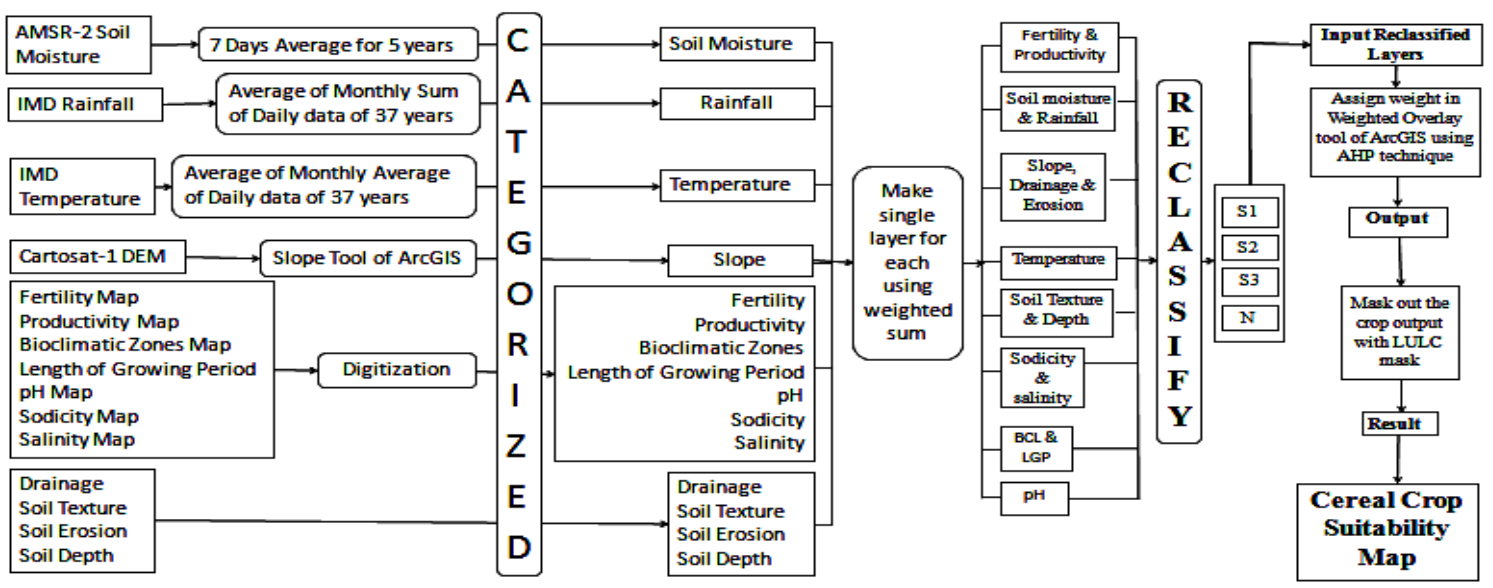

Figure 3. Methodology flow-chart

\section{RESULTS \& DISCUSSION}

Uttar Pradesh is the back bone of Indian agriculture. It plays a significant role in fulfilling the food requirement of the country. The study indicates that; Uttar Pradesh has the huge potential for the cereal crop cultivation. To find out the suitability map for cereals, each criteria were reclassified into three or four classes as S1 for highly suitable S2 for moderately suitable and $\mathrm{S} 3$ for low and $\mathrm{N}$ for not suitable. Above fifteen reclassified map were used for weighted overlay process of spatial analysis tool, using the weightage found by AHP technique to generate cereal crop suitability maps of Uttar Pradesh. The suitability map was identified in four categories as high, moderate, low and not suitable / non-agricultural lands.

This study was done for different types of cereal crops for study area such as rice, wheat, sorghum, maize and pearl millet / bajra. The following figure 4 represents the spatial distribution of cereal crops suitability level in Uttar Pradesh as; high, moderate, low and non-agricultural / not suitable lands for cereals, whereas Table:11 shows the suitable area in hectare and percentage over agricultural area for different cereal crops under high, moderate and low suitable conditions.
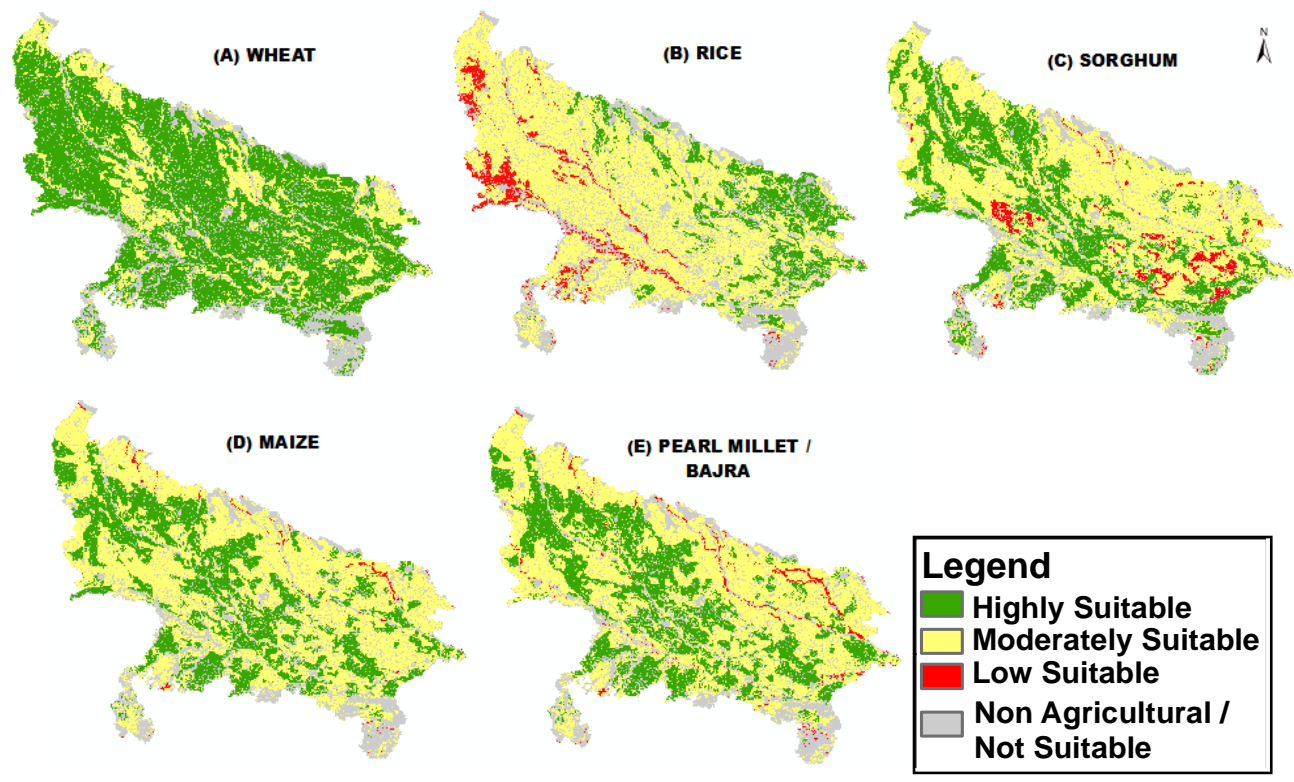

Figure 4. Cereal Crop Suitability Map of Uttar Pradesh (A) Wheat, (B) Rice, (C) Sorghum, (D) Maize, (E) Pearl Millet / Bajra

\begin{tabular}{|c|c|c|c|c|c|}
\hline $\begin{array}{c}\text { CEREAL } \\
\text { CROPS }\end{array}$ & RICE & WHEAT & SORGHUM & MAIZE & $\begin{array}{c}\text { PEARL } \\
\text { MILLET/ } \\
\text { BAJRA }\end{array}$ \\
\hline $\begin{array}{c}\text { Suitability } \\
\text { classes }\end{array}$ & Area (ha) & $\begin{array}{c}\text { Area } \\
\text { (ha) }\end{array}$ & Area (ha) & Area (ha) & Area (ha) \\
\hline $\begin{array}{c}\text { Highly } \\
\text { Suitable }\end{array}$ & $\begin{array}{c}\mathbf{2 5 , 5 1 , 3 5 0} \\
(\mathbf{1 5 \%})\end{array}$ & $\begin{array}{c}\mathbf{1 2 7 4 1 1 0 0} \\
(\mathbf{7 4 . 4 3 \%})\end{array}$ & $\begin{array}{c}\mathbf{5 6 , 3 3 , 2 3 0} \\
(\mathbf{3 2 \%} \%\end{array}$ & $\begin{array}{c}\mathbf{6 0 , 2 1 , 5 0 0} \\
\mathbf{( 3 5 \% )}\end{array}$ & $\begin{array}{c}\mathbf{6 0 , 4 8 , 8 7 5} \\
\mathbf{( 3 4 . 8 6 \%}\end{array}$ \\
\hline
\end{tabular}

\begin{tabular}{|c|c|c|c|c|c|}
\hline $\begin{array}{c}\text { Moderately } \\
\text { Suitable } 23,10,125 \\
(\mathbf{7 1 \%})\end{array}$ & $\begin{array}{c}4510925 \\
(26 \%)\end{array}$ & $\begin{array}{c}1,09,32,300 \\
(63 \%)\end{array}$ & $\begin{array}{c}1,10,95,600 \\
(\mathbf{6 4 \%})\end{array}$ & $\begin{array}{c}\mathbf{1 , 0 8 , 6 2 , 9 5 0} \\
(\mathbf{6 2 . 6 1 \%})\end{array}$ \\
\hline $\begin{array}{c}\text { Low } \\
\text { Suitable }\end{array}$ & $\begin{array}{c}\mathbf{1 0 , 7 6 , 4 0 0} \\
(6 \%)\end{array}$ & $\begin{array}{c}\mathbf{5 5 2 5} \\
(\mathbf{0 . 0 3 2} \%)\end{array}$ & $\begin{array}{c}\mathbf{6 , 9 1 , 9 5 0} \\
(4 \%)\end{array}$ & $\begin{array}{c}\mathbf{1 , 4 0 , 4 5 0} \\
(1 \%)\end{array}$ & $\begin{array}{c}\mathbf{3 , 4 5 , 7 2 5} \\
(1.99 \%)\end{array}$ \\
\hline
\end{tabular}

Table 11. Suitable area of Cereals over agricultural area 
By calculating the area of land use land cover mask Uttar Pradesh has $79.32 \%$ cultivable area. According to the generated crop suitability map for cereal crops, it was determined that; for Rice- only $15 \%$ agricultural land is highly suitable of the total cultivable land. The eastern Uttar Pradesh consists as the most favourable for rice cultivation. Whereas more of the agricultural area of the state $(71 \%)$ is moderately suitable for rice cultivation. And the low suitable area is only $6 \%$ of total cultivable land which is in the part of western Uttar Pradesh, Bank of River and in Bundelkhand region of the study area.

For Wheat- there are most of the agricultural region $(74.43 \%)$ is highly suitable, moderately suitable region is comparatively lower than highly suitable area ( $26 \%$ of total cultivable land) it is around $1 / 3$ of highly suitable regions. And the low suitable area is negligible its only $0.032 \%$ of total cultivable area of the state.

For Sorghum- there are most of the agricultural region (63\%) is moderately suitable for sorghum cultivation. The highly suitable area for sorghum is half (32\% of the total agricultural region) of the moderately suitable region. And the low suitable region is only $4 \%$ of the total cultivable land.

For Maize- there are around 2/3 of the agricultural region (64\%) is moderately suitable for sorghum cultivation. The $35 \%$ area of total cultivable area is highly suitable. And the low suitable area is negligible its only $1 \%$ of total cultivable area of the state.

For Pearl millet/Bajra- There are $34.86 \%$ highly suitable. The most of the state cultivable area is $(62.61 \%)$ moderately suitable. And only $1.99 \%$ cultivable area is low suitable for Bajra. And rest of the area is not suitable for the cereal crops.

By the above description it can be see that the study area has the huge potential for the cereal crops.

\section{CONCLUSION \& RECOMMENDATIONS}

The main objective of this research work was to generate the crop suitability map of Uttar Pradesh. It is believed that this map will provide better information to the farmers to select their cropping pattern according to the high, moderate low and not suitable region.

The weighted overlay method in spatial analysis technique was used to locate the suitable location for different-different crops. The study was carried out based on soil physical and chemical properties, topography, climate and land use and land cover of the study area. The methodology of MCDM in AHP technique was used which provided a guide for decision making about taking consideration of different information related to requirements of selected crops for assigning the weightage on the basis of their priorities. Additionally, the results of this study could be useful for other investigators who could use these results for diverse studies for different areas, such as planners, policy makers, government organizations, etc.

Research of the future should further examine the suitability analysis taking more factors which will improve the accuracy of result, such as;

- $\mathrm{CACO}_{3}$ content,

- Organic content,

- Socio-economic condition of farmers.

\section{REFERENCES}

Anushiya1, J. \& Illeperuma, I. A. (2016). "Suitability Analysis for Paddy Cultivation Sites Using A Multi Criteria Evaluation and Gis Approach Case Study: Imbulpe Ds Division In Sri Lanka"Asian Association on Remote Sensing

Ayehu, Getachew T., and Solomon A. Besufekad. (2015) "Land suitability analysis for rice production: A GIS based multi-criteria decision approach:." American Journal of Geographic Information System 4.3 95-104.

Bhaskar, K. S., and S. T. Gaikwad. "Anantha Rao and Rao, A.(1996)." Soil-site suitability for wheat-a case study. Agropedology 6: 89-94.

FAO (1976). A Framework for Land Evaluation. Soils Bull. 32, FAO, Rome. FAO (1983). Guidelines Land Evaluation for rainfed agriculture.

Gill, Khem Singh. (1991). "Pearlmillet and its Improvement." Indian Council of Agricultural Research, New Delhi. 297 pp.

Girl, J.D. Shyampur, R.L. and Sehgal, J.L. (1994). Soil-site suitability for maize in Banswara District, Rajsthan. Agropedology, 4:75-79.

https://en.wikipedia.org/wiki/Uttar_Pradesh (accessed on 23/04/2017)

https://www.youtube.com/channel/UCnDfmcUyhgJp6xC1 LmBLfUg $\backslash$ (accessed on 23/04/2017)

http://www.imdpune.gov.in (ncc@imd.gov.in) (National Climate Centre India Meteorological Department)

Naidu, L. G. K., et al. (2006) "Manual soil-site suitability criteria for major crops." NBSS Publ 129: 118.

Patel, Jayesh H., and Markand P. Oza. 8 (2014) "Deriving crop calendar using NDVI time-series:" The International Archives of Photogrammetry, Remote Sensing and Spatial Information Sciences 40. 869.

Pramanik, Malay Kumar (2016) "Site suitability analysis for agricultural land use of Darjeeling district using AHP and GIS techniques." Modeling Earth Systems and Environment 2.2: 56.

Saaty, T.L. (1980). The Analytic Hierarchy Process. McGraw-Hill, New York.

Saaty, Thomas L. (1978) "Exploring the interface between hierarchies, multiple objectives and fuzzy sets." Fuzzy sets and systems 1.1: 57-68.

Sharma, S. (2006). Soil Moisture Estimation Using Active And Passive Microwave Remote Sensing Techniques. M. Tech Thesis. IIRS Dehradun.

sYalew, Seleshi G., et al. (2016) "Land suitability analysis for agriculture in the Abbay basin using remote sensing, GIS and AHP techniques." Modeling Earth Systems and Environment2.2: 101.

By using these parameters the result will be more refine. 